Mitteilungen des Verlags

16 Titeländerung: Viszeralmedizin

Editorials

17 Chirurgische Gastroenterologie Interdisziplinär wird zu Viszeralmedizin

Klar, E. (Rostock); Mössner, J. (Leipzig); Lohse, A.W. (Hamburg)

18 Lokale Therapie von Frühkarzinomen: «Progress to something better or to something new?»

Anthuber, M. (Augsburg); Tannapfel, A. (Bochum);

Ell, C. (Wiesbaden)

278 Hepatozelluläres Karzinom

Galle, P.R. (Mainz); Jonas, S. (Leipzig)

3130 NOTES - warum?

Meier, P.N. (Hannover); Fuchs, K.-H. (Frankfurt/M.)

4178 Chronisch-entzündliche Darmerkrankungen Sinn und Nutzen der Zusammenarbeit von Gastroenterologen und Chirurgen Kruis, W. (Köln); Buhr, H.-J. (Berlin)

Hauptthemen

Submukosakarzinome des Gastroinestinaltraktes: Immer Operation oder auch endoskopische Therapie?

Herausgeber: J. Anthuber, Augsburg; A. Tannapfel, Bochum; C. EII, Wiesbaden

111 Histopathologische Besonderheiten von Submukosakarzinomen

Tannapfel, A. (Bochum)

114 Ösophagus: Pro Endoskopie Manner, H.; May, A.; Pech, O.; Ell, C. (Wiesbaden)

117 Ösophagus-Frühkarzinome: Pro Chirurgie Bogoevski, D.; Kutup, A.; König, A.M.; Busch, C.T.H.; Izbicki, J.R. (Hamburg)

121 Submukosal invasives Magenfrühkarzinom: Pro Endoskopie Nordmann, T.; Neuhaus, H. (Düsseldorf)

126 Submukosakarzinome des Gastrointestinaltrakts Magen: Stadiengerechte Therapie Schuhmacher, C.; Reim, D.; Novotny, A.; Friess, H. (München)
134 Kolon und Rektum: Pro Endoskopie Schmitt, W. (München)

140 Kolon: Pro Chirurgie

Weber, T.; Link, K:H: (Wiesbaden)

146 Submukosakarzinome des Rektums: Lokale Therapie - pro Chirurgie

Göhl, J.; Merkel, S. (Erlangen)

\section{Hepatozelluläres Karzinom}

Herausgeber: P.R. Galle, Mainz; S. Jonas, Leipzig

279 Hepatozelluläres Karzinom: Diagnostischer Algorithmus

Geißler, M.; Krämer, S. (Esslingen)

285 Hepatozelluläres Karzinom: Transkutane lokal ablative Verfahren und/oder transarterielle

Chemoembolisation

Allgaier, H.-P.; Galandi, D. (Freiburg i.Br.)

290 Hepatozelluläres Karzinom - laparoskopische und konventionell-chirurgische Verfahren

Mirow, L. (Mittweida); Bader, F.G. (Lübeck); Kunath, H.; Schnee, S. (Mittweida); Roblick, U.; Bruch, H.-P-; Hildebrand, P. (Lübeck)

297 Medikamentöse Therapie des hepatozellulären Karzinoms

Greten, T.F. (Hannover)

Standortbestimmung und Entwicklung: NOTES

Herausgeber: K.-H. Fuchs, Frankfurt/M.; P.N. Meier, Hannover (Hrsg.)

3134 Flexibel oder starr - wie soll die Zukunft aussehen? Kaehler, G. (Mannheim)

3139 NOTES - gastroenterologische Perspektive von Renteln, D.; Caca, K (Ludwigsburg)

3144 Chirurgische Perspektiven in der NOTES-Entwicklung Bernhardt, J.; Steffen, H.; Wießner, R.; Schober, H.-C.; Ludwig, K. (Rostock)

3149 Standortbestimmung und Entwicklung: NOTES welcher Zugang? von Delius, S.; Wilhelm, D.; Feußner, H.; Meining, A. (München)

3157 NOTES - internationaler Stand Hochberger, J.; Menke, D. (Hildesheim); Köhler, P. (Mariensee); Lamadé, W. (Stuttgart) 
Chronisch Entzündliche Darmerkrankungen

Herausgeber: W. Kruis, Köln; H.-J. Buhr, Berlin

4179 Kurzfassung der aktualisierten S3-Leitlinie der Deutschen Gesellschaft für Verdauungs- und Stoffwechselkrankheiten (DGVS) und des Kompetenznetzes Darmerkrankungen zur Diagnostik und Behandlung des Morbus Crohn

Hoffmann, J.C. (Ludwigshafen); Bokemeyer, B. (Minden); Preiß, J.C.; Siegmund, B. (Berlin); Stange, E.F. (Stuttgart); Zeitz, M. (Berlin)

4187 Kurzdarstellung der Therapierichtlinien zur

Colitisulcerosa

Rogler, G. (Zürich)

4194 Chirurgische und medikamentöse Therapie Morbus-

Crohn-assoziierter Fisteln

Kroesen, A.J.; Holtmeier, W. (Köln)

4199 Therapie chronisch entzündlicher Darmerkrankungen: Konservative Therapie - Immunmodulatoren Herrlinger, K. (Stuttgart)

4204 Konservative Therapie: «Step Up» oder «Step Down»? Emmrich, J. (Rostock)

4209 Lebensqualität nach operativer Therapie der Colitisulcerosa und Morbus Crohn Heuschen, G.; Heuschen, U. (Limburg/Lahn)

4215 Karzinomrisiko bei chronisch-entzündlichen Darmerkrankungen: Über- oder unterschätzt? Bielecki, C.; Stallmach, A, (Jena)

4219 OP-Indikation: Wie oft zuviel und zu frïh operiert? Manthey, C.; Pace, A. (Hamburg)

4225 OP-Indikation: Wird oft zu spät operiert? Sicht des Viszeralchirurgen Plusczyk, T.; Schilling, M. (Homburg/Saar)

Interdisziplinäres Gespräch

152 Submukosakarzinome des Gastrointestinaltrakts: Immer Operation oder auch endoskopische Therapie? Kähler, G. (Discussion Leader) (Mannheim)

2100 Hepatozelluläres Karzinom Lang, H. (Mainz) (Discussion Leader)

3163 Standortbestimmung und Entwicklung: NOTES Feußner, H. (München) (Discussion Leader)

4230 Chronisch-entzündliche Darmerkrankungen Stange, E. (Stuttgart) (Gesprächsleiter)
Übersichtsarbeiten

157 Aktuelle Standards in der Behandlung der nekrotisierenden Pankreatitis - was ist Evidenzbasiert?

Schleicher, C.; Torge Meles, S.; Colombo-Benkmann, M.; Senninger, N. (Münster)

2105 Multimodale Therapie von Kolon- und Rektumkarzinomen: Qualitätsparameter Link, K.-H. (Wiesbaden); Kornmann, M. (Ulm); Mann, M. (Wiesbaden); Bittner, R. (Stuttgart)

Fallberichte

4234 Double-Duct-Sign in der endoskopisch retrograden Cholangiopankreatikographie ohne sichtbaren Tumor in der Querschnittsbildgebung - alt, aber noch gut, um ein Pankreaskarzinom zu diagnostizieren?

Groth, S.; Kutup, A.; Buhk, J.-H.; Marx, A.; Adam, G.; Izbicki, J.R.; Sauter, G.; Rösch, T. (Hamburg)

Buchbesprechungen

$\mathbf{1} 67,2118$

News / Ticker

$\mathbf{1}$ 65, 2 119, 3169,4238

Tagungen und Kongresse

1 70, 2 121, 3 170, 4239

4242 Autorenverzeichnis Band 25, 2009

4243 Sachwortverzeichnis Band 25, 2009 